\title{
Securing The Predicting Data Using Rjb20 Algorithm
}

\author{
R. Jaichandran ${ }^{1}$, S. Muthuselvan ${ }^{3}$, P. Malli Karjun Reddy ${ }^{4}$, \\ L. Sriharinath Reddy ${ }^{5}$, And Kona Ricky Martin 6 \\ 1,3,4,5,6 Department Of Computer Science And Engineering \\ AarupadaiVeeduInstitute OfTechnology \\ VinayakaMission'S Research Foundation \\ Paiyanoor-603 104,Tamil Nadu, India. \\ ${ }^{1}$ rjaichandran@avit.ac.in, ${ }^{3}$ muthuselvan@avit.ac.in, \\ 4jackson.malli08@gmail.com \\ ${ }^{5}$ harinathsri20@gmail.com, ${ }^{6}$ rickylovely777@gmai.com
}

\author{
Dr. Avinash Sharma ${ }^{2 * *}$ \\ ${ }^{2}$ Professor, CSE Department, M.M. Deemed to be University, Mullana, Haryana, India, \\ 133207 \\ asharma@mmumullana.org \\ Corresponding Author: Dr. Avinash Sharma ${ }^{2 * *}$
}

\begin{abstract}
The current world is information world; without this information can't make due in present stage. This information created more from web- based media; this media information is public information; This public infor- mation did not have well security; so we applying the proposed method and it has 2 steps; 1 . Addition property of the matrix;2. Perfect numbers swapped. The proposed method gives well security while comparing with Salsa method.
\end{abstract}

Key words: Commutative Property, RJB20, Salsa, Encryption, Decryption.

\section{INTRODUCTION}

The current world is information world; without this information can't make due in present stage. This information created more from web-based media; this media information is public information; This public information did not have well security; so to conquer this matter we apply the Salsa strategy. This strategy effectively hack the information from the programmers. The additional rotations XOR for ChaCha is fault attack [1]. This author is used new hash concept for key guessing and halting condition [2]. Author was introduced thw bricklayer attack for analysis of ChaCha [3]. They mainly focuse the security for Double A [4]. They made new design for secure fast and flexible algorithm [5]. SRB18 method used to give security for data [6]. SRB21 method used to give security for data [7]. CBB21 method used to provide security for data [8]. CBB22 method used to provide security for data [9]. Introducedthenew method RJB20 ( RajaprakashJaichandran and BagathBasha) 20 for this problem. 


\section{METHODS}

Commutative property of addition (CP): This property discuss in Table 1 and Table2.

\section{ENCRYPTION}

"A is analyzed matrix"; and "B is secret matrix". [10]

"Equation (1)"

$$
\begin{gathered}
“ \mathrm{~A}=\left(\begin{array}{lll}
1 & 2 & 3 \\
4 & 5 & 6 \\
7 & 8 & 9
\end{array}\right), " \mathrm{~B}=\left(\begin{array}{lll}
2 & 4 & 3 \\
5 & 6 & 1 \\
7 & 9 & 8
\end{array}\right), \\
" \mathrm{CP}=\left(\begin{array}{lll}
1 & 2 & 3 \\
4 & 5 & 6 \\
7 & 8 & 9
\end{array}\right) "+\left(\begin{array}{lll}
2 & 4 & 3 \\
5 & 6 & 1 \\
7 & 9 & 8
\end{array}\right), \\
\mathrm{CP}=\left(\begin{array}{ccc}
3 & 6 & 6 \\
9 & 11 & 7 \\
14 & 17 & 17
\end{array}\right)
\end{gathered}
$$

Equations “(2)"

"Pair-1 (3, 0)"

$$
\mathrm{PN}=\left(\begin{array}{ccc}
9 & 6 & 6 \\
3 & 11 & 7 \\
14 & 17 & 17
\end{array}\right)
$$

"Pair-2 (1, 0)"

$$
P N=\left(\begin{array}{ccc}
6 & 9 & 6 \\
3 & 11 & 7 \\
14 & 17 & 17
\end{array}\right)
$$

"Pair-3 (4, 0)" "Pair-4 (2, 0)"'Pair-5 (4, 5)"'Pair-6 (3, 0)"'Pair-7 (4, 0)"

"Pair-8 (5, 1)"

$$
P N=\left(\begin{array}{ccc}
7 & 6 & 11 \\
6 & 3 & 9 \\
14 & 17 & 17
\end{array}\right)
$$

"Pair-9 (0, 4)"

$$
\mathrm{EM}=\left(\begin{array}{ccc}
3 & 6 & 11 \\
6 & 7 & 9 \\
14 & 17 & 17
\end{array}\right)
$$


TABLE 1. RJB20 Secure Encryption

\begin{tabular}{|c|c|}
\hline STEPS & RJB20 SECURE ENCRYPTION \\
\hline $\mathrm{i}$ & " The data analyzed from social data". \\
\hline ii & "The data will form a matrix" \\
\hline iii & $\begin{array}{l}\text { "The commutative property (CP) concept applied in matrix CP" } \\
" C P=A+B=B+A " \text { (1) }\end{array}$ \\
\hline iv & "Prime numbers in the Matrix A". \\
\hline $\mathrm{v}$ & $" P N=\left(e^{k-1}\right)\left(e^{k}\right)-1 "(\mathbf{2})$. \\
\hline vi & $\begin{array}{l}\text { "EM=PN" } \\
\text { where EM is EncryptedMatrix }\end{array}$ \\
\hline
\end{tabular}

TABLE 2. RJB20 Secure Decryption

\begin{tabular}{|l|l|}
\hline STEPS & RJB20 SECURE DECRYPTION \\
\hline i & "Prime numbers in the Matrix EM". \\
\hline ii & $P N=\left(d^{k-1}\right)\left(d^{k}\right)-1(\mathbf{3})$ \\
\hline iii & $\begin{array}{l}D M 1=P N \\
\text { where DM1 is Decrypted Matrix 1 }\end{array}$ \\
\hline iv & $\begin{array}{l}\text { "Minus the secret key matrix B with the matrix DM1". } \\
D M 2=D M 1-B \text { (4) } \\
\text { where DM2 is Decrypted Matrix 2 }\end{array}$ \\
\hline
\end{tabular}

Equation "(3)"

"Pair-1 (4, 0)"

$$
\mathrm{PN}=\left(\begin{array}{ccc}
7 & 6 & 11 \\
6 & 3 & 9 \\
14 & 17 & 17
\end{array}\right)
$$

"Pair-2 (1, 5)"

$$
\mathrm{PN}=\left(\begin{array}{ccc}
7 & 9 & 11 \\
6 & 3 & 6 \\
14 & 17 & 17
\end{array}\right)
$$

"Pair-3 (0, 4)"'Pair-4 (0, 3)"'Pair-5 (5, 4)"'Pair-6 (0, 2)"'Pair-7 (0, 4)"

"Pair-8 (0, 1)"

$$
\mathrm{PN}=\left(\begin{array}{ccc}
9 & 6 & 6 \\
3 & 11 & 7 \\
14 & 17 & 17
\end{array}\right)
$$

"Pair-9 (0, 3)" 


$$
\begin{aligned}
\mathrm{DM} 1 & =\left(\begin{array}{ccc}
3 & 6 & 6 \\
9 & 11 & 7 \\
14 & 17 & 17
\end{array}\right) \\
\text { "Equation (4)". "DM2 } & =\mathrm{DM} 1-\mathrm{B} " \\
\mathrm{DM} 2 & =\left(\begin{array}{lll}
1 & 2 & 3 \\
4 & 5 & 6 \\
7 & 8 & 9
\end{array}\right)
\end{aligned}
$$

\section{CONCLUSION}

Prediction of an environmental parameter is possible using sensing[16-20] or IoT [21-23] mechanism but prediction of security is based on features of data or Big Data[24,25]. Different kinds of prediction possible using various techniques including ML[26,27].

The current world is information world; without this information can'tmake due in present stage. This information created more from web-based media; this media information is public information; This public information did not have well security; so we applied the RJB20 method and it has 2 steps; 1. Ad- dition property of the matrix;2. Perfect numbers swapped. The RJB20 method giveswellsecuritywhilecomparingwithSalsamethod

\section{REFERENCES}

[1] P.A.BABUANDJ.J.THOMAS:APRACTICALFAULTATTACKONARXlikeCipherswithACASE Study on CHACHA20, Wo. onFa. Di. andTo. inCr. (2017),33-40.

[2] S. V. D. KUMAR, S. PATRANABIS, J. BREIER, D. MUKHOPADHYAY, S. BHASIN, CHATTOPADHYAY,ANDA.BAKS:Freestyle, ARANDOMIZEDversionofCHACHAforres isting offline brute-force ANDDICTIONARY ATTACKS, IE. tr. on In. Fo. and Se.(2018).

[3] ALEXANDRE ADOMNICAI, JACQUES J. A. FOURNIER, AND LAURENT MASSON: Brick- LAYERATTACK:ASIDECHANNELANALYSISonthe ChaCHaQUARTERRound,Pro.in Cry.Ind.,Lec. Not. in Com. Sci., Spr.65-84.

[4] BODHISATWA MAZUMDAR, SK SUBIDH ALI AND OZGUR SINANOGLU: Power ANALYSISATTACKSONARX:AnAPPLICATIONTOSALSA20,On.Tes.Sym.IEE.(2015),40-43.

[5] C. WATT, J. RENNER, N. POPESCU, S. CAUligi, AND D. STEFAN: CTWASM:TypeDrivenSecure CRYPTOGRAPHYfortheWebEcosystem,Pr.ACMPr.La.PO.(2019),77:1-77:29.

[6] C. BAGATH BASHA, S. RAJAPRAKASH: ENHANCING The Security Using SRB18 Method of Embedding Computing, Mir. andMic 103125,(2020).

[7] C. B. BASHA, S. RAJAPRAKASH:SecuringTwitterDATAUsingSrb21PHASEIMethodology,Int.Jou.ofSci.andTec.Res.8(12)(2019),1952-1955.

[8] C. B. BASHA, S. RAJAPRAKASH: Applying The CBB21 PHASE 2 Method For Securing Twitter ANALYSED DATA, Ad. In Ma. : Sc. Jo. 9(3) (2020),1085-1091.

[9] C. B. BASHA, S. RAJAPRAKASH, V. V. A. HARISH, M. S. KRISHNA, K. PRABHAS:SecuringTwitterANALYSEDDATAUsingCBB22Algorithm,Ad.InMa.:Sc. Jo. 9(3) (2020), 1093-1100.

[10] C.B.BASHA,K.SOMASUNDARAM:ACOMPARATIVEStudyofTwitterSentimentANALysis Using MACHINELEARNING Algorithms in Big DATA, Int. Jou. of Rec. Tec. and Eng. 8(1) (2019),591-599.

[11] Somasekar, J. \& Sharma, A. \& Reddy, N. \& Reddy, Y.. (2020). IMAGE ANALYSIS FOR AUTOMATIC ENUMERATION OF RBC INFECTED WITH PLASMODIUM 
PARASITES-IMPLICATIONS FOR MALARIA DIAGNOSIS. Advances in Mathematics: Scientific Journal. 9. 1221-1230. 10.37418/amsj.9.3.48.

[12] A. SHARMA AND J. SOMASEKAR "Contrast Image Construction Technique for Medical Imaging" published in Advances in Mathematics: Scientific Journal (Adv. Math., Sci. J.) vol9-no-6-2020 (pp 3325-3329)

[13] Rohini Goel, Avinash Sharma, and Rajiv Kapoor, "Object Recognition Using Deep Learning" published in Journal of Computational and Theoretical Nanoscience Vol. 16, 40444052, 2019

[14] Santosh, Mamta \& Sharma, Avinash. (2019). A Proposed Framework for Emotion Recognition Using Canberra Distance Classifier. Journal of Computational and Theoretical Nanoscience. 16. 3778-3782. 10.1166/jctn.2019.8250.

[15] Mamta Santosh, Avinash Sharma, "Facial Expression Recognition using Fusion of LBP and HoG Features" published in International Journal of Innovative Technology and Exploring Engineering (IJITEE) ISSN: 2278-3075, Volume-8 Issue-8 June, 2019

[16] Varsha, N. Kumar, Energy Efficient TABU Optimization Routing Protocol for WSN,IngenieríaSolidaria, Universidad Cooperativa de Colombia, Issue- 33, July 2020.

[17] G.Arora, A.Kumar, Versha, N.Kumar, "Swarm Intelligence based QoS optimized routing in WSN", Test Engineering \& Management, Vol.-82, 2020.pp-12880-12885.

[18] Varsha, M. B., Kumar, M., \& Kumar, N. Hybrid TABU-GA Search For Energy Efficient Routing In WSN. International Journal of Recent Technology and Engineering (IJRTE) ISSN: 2277-3878, Volume-8 Issue-4, November 2019.P.-3250-3256.

[19] Varsha, M. B., Kumar, M., \& Kumar, N. Development of QoS optimized routing using Artificial bee colony and TABU-GA with a mobile base station in Wireless Sensor Network, International Journal of Innovative Technology and Exploring Engineering (IJITEE) ISSN: 2278-3075, Volume-9 Issue-1, November 2019.

[20] Awadhesh Kumar Maurya,Varsha, Neeraj, Ajay Kumar, Neeraj Kumar, "Improved chain based cooperative routing protocol in wsn", FEST, Journal of Physics: Conference series, IOP Publishing, 1478,1-8, 13/05/2020.

[21] N. Kumar, A. Agrawal,R. A. Khan, "METHWORK: An Approach for Ranking in Research Trends with a Case Study for IoET, Recent advances in Computer Science and Communication (formerly Recent Patents on Computer Science), 2019.

[22] Neeraj Kumar; PareshGoyal; GayatriKapil; AlkaAgrawal; Raees A Khan, "Flood Risk Finder for IoT based Mechanism using Fuzzy Logic", Materials Today: Proceedings, Elsevier, 2020.

[23] Kumar, Neeraj, AlkaAgrawal, and R. A. Khan. "Cost estimation of cellularly deployed IoTenabled network for flood detection." Iran Journal of Computer Science, issue 2, no. 1 (2019), Springer Nature: 53-64.

[24] V. Velvizhi; Satish R Billewar; GauravLondhe; PravinKshirsagar; Neeraj Kumar, "Big Data for Time Series and Trend Analysis of Poly Waste Management in India", Materials Today: Proceedings, Elsevier, 2020.

[25] G. Arora, A. K. Maurya,N. Kumar, A. K. Mishra, "Application of big data generated by IoT environment for HealthCare using Voice Recognition", International journal of research in engineering, IT and Social Sciences, vol.-08, issue-11, November 2018, page. 132-136.

[26] Manoj Diwakar, AmrendraTripathi, Kapil Joshi, MinakshiMemoria , Prabhishek Singh, Neerajkumar, "Latest Trends on Heart Disease Prediction using Machine Learning and Image Fusion", Materials Today: Proceedings,Elsevier, 2020.

[27] ParthWadhwa; Aishwarya; AmrendraTripathi; Prabhishek Singh; Manoj Diwakar; Neeraj Kumar, "Predicting the Time Period of Extension of Lockdown due to Increase in Rate of COVID-19 Cases in India using Machine Learning", Materials Today: Proceedings Elsevier, 2020 . 
European Journal of Molecular \& Clinical Medicine ISSN 2515-8260 Volume 07, Issue 06, 2020 